\title{
THE THURSTON NORM AND TWISTED ALEXANDER POLYNOMIALS
}

\author{
STEFAN FRIEDL AND STEFANO VIDUSSI
}

\begin{abstract}
Using recent results of Agol, Przytycki-Wise and Wise we show that twisted Alexander polynomials detect the Thurston norm of any irreducible 3manifold which is not a closed graph manifold.
\end{abstract}

\section{INTRODUCTION AND MAIN RESULTS}

Let $N$ be a 3-manifold. (Throughout the paper, unless otherwise stated, we will assume that all 3-manifolds are orientable, connected and that they have either empty or toroidal boundary.) Given a surface $\Sigma$ with connected components $\Sigma_{1}, \ldots, \Sigma_{k}$ its complexity is defined to be

$$
\chi_{-}(\Sigma)=\sum_{i=1}^{k} \max \left\{-\chi\left(\Sigma_{i}\right), 0\right\} .
$$

Given a 3-manifold $N$ and $\phi \in H^{1}(N ; \mathbb{Z})$ the Thurston norm is defined as

$$
x_{N}(\phi):=\min \left\{\chi_{-}(\Sigma) \mid \Sigma \subset N \text { properly embedded and dual to } \phi\right\} .
$$

Thurston [Th86] showed that $x_{N}$ is a seminorm on $H^{1}(N ; \mathbb{Z})$. We say that a class $\phi \in H^{1}(N ; \mathbb{Z})=\operatorname{Hom}\left(\pi_{1}(N), \mathbb{Z}\right)$ is fibered if there exists a fibration $p: N \rightarrow S^{1}$ such that $\phi=p_{*}: \pi_{1}(N) \rightarrow \mathbb{Z}$. We refer to Section 2 for more information on the Thurston norm and fibered classes.

Given a 3-manifold $N$, a class $\phi \in H^{1}(N ; \mathbb{Z})$, and a representation $\alpha: \pi_{1}(N) \rightarrow$ $\mathrm{GL}(k, \mathbb{C})$ we denote by $\tau(N, \phi, \alpha) \in \mathbb{C}(t)$ the corresponding twisted Reidemeister torsion, whose definition we summarize in Section 3.1. This invariant was first introduced by Lin [Lin01] and Wada [Wa94] using slightly different normalizations. In the literature $\tau(N, \phi, \alpha)$ is also sometimes referred to as the twisted Alexander polynomial of $(N, \phi, \alpha)$. Our approach in defining $\tau(N, \phi, \alpha)$ follows the point of view taken in [KL99] and FK06.

Given $p(t) \neq 0 \in \mathbb{C}\left[t^{ \pm 1}\right]$ we can write $p(t)=\sum_{i=r}^{s} a_{i} t^{i}$ with $a_{r} \neq 0$ and $a_{s} \neq 0$ and we define

$$
\operatorname{deg}(p(t)):=s-r
$$

Date: April 25, 2018.

1991 Mathematics Subject Classification. 57M27.

S. Vidussi was partially supported by NSF grant DMS-0906281. 
We extend this definition to $\operatorname{deg}(0):=0$. Furthermore, given $f(t) \in \mathbb{C}(t)$ we can write $f(t)=\frac{p(t)}{q(t)}$ with $p(t), q(t) \in \mathbb{C}\left[t^{ \pm 1}\right]$, and we define

$$
\operatorname{deg}(f(t)):=\max \{0, \operatorname{deg}(p(t))-\operatorname{deg}(q(t))\} .
$$

Note that the degree of $f(t)$ is well--defined.

In [FK06, Theorems 1.1 and 1.2] the first author and Taehee Kim proved the following theorem:

Theorem 1.1. Let $N$ be a 3-manifold, $\phi \in H^{1}(N ; \mathbb{Z})$ non-zero and $\alpha: \pi_{1}(N) \rightarrow$ $G L(k, \mathbb{C})$ a representation, then

$$
\frac{1}{k} \operatorname{deg}(\tau(N, \phi, \alpha)) \leq x_{N}(\phi) .
$$

Furthermore equality holds if $\phi$ is fibered.

It is a natural question to ask whether there exists a representation such that (11) becomes an equality for all $\phi$. Computational evidence towards an affirmative answer was given in [FK06, FK08] and [DFJ11]. Using recent work of Agol Ag08, Ag12, Przytycki-Wise [PW12] and Wise [Wi12] (see Section 5.2 for details) we can now prove that that this is indeed the case for most 3-manifolds:

Theorem 1.2. Let $N$ be an irreducible 3-manifold which is not a closed graph manifold. Then there exists a unitary representation $\alpha: \pi_{1}(N) \rightarrow U(k)$ such that

$$
\frac{1}{k} \operatorname{deg}(\tau(N, \phi, \alpha))=x_{N}(\phi) \text { for any } \phi \in H^{1}(N ; \mathbb{Z}) \backslash\{0\} .
$$

Furthermore $\alpha$ can be chosen to factor through a finite group.

Remark. (1) In Corollary 5.10 we will prove a closely related result which shows that under the same assumption on $N$ there exists a unitary representation such that the corresponding twisted Alexander norm introduced in FK08 (which generalizes work of McMullen [Mc02] and Turaev [Tu02a]) also detects the Thurston norm.

(2) It was shown by many authors (see [Ch03, GM03, GKM05, FK06, Pa07, Kiy08, FV10]), at different levels of generality, that twisted Alexander polynomials give obstructions to a 3-manifold being fibered. In [FV11a. (see also [FV11b]) the authors showed that in fact twisted Alexander polynomials detect fibered 3-manifolds. The fiberedness criterion of [FV11a] can be greatly strengthened using the recent work of Wise. We refer to [FV11c] for details.

(3) Some of the ideas of this paper were also used by the authors in FV11d to study genus minimizing surfaces in $S^{1}$-bundles over closed 3-manifolds.

We conclude with a quick summary of two applications to our results:

(1) Let $N$ be an irreducible 3-manifold which is not a closed graph manifold. Theorem 1.2 gives a new algorithm for determining the Thurston norm. We refer to Section 6 for details. 
(2) In [FSW12] we will use Theorem 1.2 to study 'splittings of knot groups along minimal edge groups' and we will furthermore use Theorem 1.2 to relate the Thurston norm to the Turaev norm (see [Tu02b]) on the first cohomology of a 2-complex.

Convention. Unless specified otherwise, all groups are assumed to be finitely generated. Furthermore we allow norms to be degenerate, i.e. we refer to seminorms as norms. By a free abelian group we always mean a non-trivial free abelian group.

Acknowledgment. We are grateful to Genevieve Walsh for a helpful conversation.

\section{The Thurston nORM}

Let $N$ be a 3 -manifold and let $\phi \in H^{1}(N ; \mathbb{Z})$. It is well-known that any class in $H^{1}(N ; \mathbb{Z})$ is dual to a properly embedded surface. Now recall that the Thurston norm of $\phi$ is defined as

$$
x_{N}(\phi):=\min \left\{\chi_{-}(\Sigma) \mid \Sigma \subset N \text { properly embedded and dual to } \phi\right\} \text {. }
$$

Thurston [Th86] showed that $x_{N}$ is a seminorm on $H^{1}(N ; \mathbb{Z})$ which thus can be extended to a seminorm on $H^{1}(N ; \mathbb{R})$ which we also denote by $x_{N}$. Thurston furthermore showed that the Thurston norm ball

$$
B(N):=\left\{\phi \in H^{1}(N ; \mathbb{R}) \mid x_{N}(\phi) \leq 1\right\}
$$

is a (possibly non-compact) finite convex polytope.

We also recall that an integral class $\phi \in H^{1}(N ; \mathbb{Z})=\operatorname{Hom}\left(\pi_{1}(N), \mathbb{Z}\right)$ is called fibered if there exists a fibration $p: N \rightarrow S^{1}$ such that $\phi=p_{*}: \pi_{1}(N) \rightarrow \mathbb{Z}$. More generally, we say that a class $\phi \in H^{1}(N ; \mathbb{R})$ is fibered if $\phi$ can be represented by a nowhere vanishing closed $1-$ form. It is well-known (see e.g. [Ti70]) that for integral classes the two notions of being fibered coincide.

Thurston [Th86] showed that there exist open top-dimensional faces $F_{1}, \ldots, F_{r}$ of $B(N)$ such that the set of fibered classes in $H^{1}(N ; \mathbb{R})$ equals the union of the open cones on $F_{1}, \ldots, F_{r}$. The faces $F_{1}, \ldots, F_{r}$ are referred to as fibered faces of $B(N)$.

Finally let $p: M \rightarrow N$ be a finite cover of degree $k$ and let $\phi \in H^{1}(N ; \mathbb{R})$. Then

$$
x_{M}\left(p^{*} \phi\right)=k \cdot x_{N}(\phi)
$$

furthermore $\phi$ is fibered if and only if $p^{*} \phi$ is fibered. We refer to [Ga83, Corollary 6.18] for a proof of (2), while the statement regarding finite covers can be proved using Stallings' theorem [St62]. 


\section{Definition and Basic properties of twisted Alexander polynomials}

3.1. Twisted Alexander polynomials. Let $N$ be a 3-manifold and let $\alpha: \pi_{1}(N) \rightarrow$ $\mathrm{GL}(k, \mathbb{C})$ be a representation. Furthermore let $\psi: \pi_{1}(N) \rightarrow F$ be a rationally surjective homomorphism to a free abelian group $F$. (Here by rationally surjective we mean that $\psi$ has finite cokernel.) We get a tensor representation

$$
\begin{aligned}
\alpha \otimes \psi: \pi_{1}(N) & \rightarrow \mathrm{GL}(k, \mathbb{C}[F]) \\
g & \mapsto \alpha(g) \cdot \psi(g) .
\end{aligned}
$$

We denote the universal cover of $N$ by $\tilde{N}$. Note that there exists a canonical left $\pi_{1}(N)$-action on the universal cover $\tilde{N}$ given by deck transformations. We consider the cellular chain complex $C_{*}(\tilde{N} ; \mathbb{Z})$ as a right $\mathbb{Z}\left[\pi_{1}(N)\right]$-module by defining $\sigma \cdot g:=$ $g^{-1} \sigma$ for a chain $\sigma$. The representation $\alpha \otimes \psi$ gives rise to a left action of $\pi_{1}(N)$ on $\mathbb{C}[F]^{k}$. We can therefore consider the $\mathbb{C}[F]$-complex

$$
C_{*}^{\psi \otimes \alpha}\left(N ; \mathbb{C}[F]^{k}\right):=C_{*}(\tilde{N} ; \mathbb{Z}) \otimes_{\mathbb{Z}\left[\pi_{1}(N)\right]} \mathbb{C}[F]^{k} .
$$

and its homology modules

$$
H_{i}^{\psi \otimes \alpha}\left(N ; \mathbb{C}[F]^{k}\right):=H_{i}\left(C_{*}(\tilde{N} ; \mathbb{Z}) \otimes_{\mathbb{Z}\left[\pi_{1}(N)\right]} \mathbb{C}[F]^{k}\right) .
$$

Since $N$ is compact and since $\mathbb{C}[F]$ is Noetherian these modules are finitely presented over $\mathbb{C}[F]$. We now define the $i$-th twisted Alexander polynomial of $(N, \psi, \alpha)$ to be the order of $H_{i}\left(N ; \mathbb{C}[F]^{k}\right)$ (see [FV10] and [Tu01] for details). We will denote it as $\Delta_{N, \psi, i}^{\alpha} \in \mathbb{C}[F]$. Throughout this paper we often write $\Delta_{N, \psi}^{\alpha}$ instead of $\Delta_{N, \psi, 1}^{\alpha}$.

Note that $\Delta_{N, \psi, i}^{\alpha} \in \mathbb{C}[F]$ is well-defined up to multiplication by a unit in $\mathbb{C}[F]$, i.e. up to an element of the form $r f$ with $r \in \mathbb{C} \backslash\{0\}$ and $f \in F$. In the following, we denote by $\mathbb{C}(F)$ the quotient field of $\mathbb{C}[F]$, and given $p, q \in \mathbb{C}(F)$ we write

$$
p \doteq q
$$

if $p$ and $q$ agree up to multiplication by an element of the form $r f$ with $r \in \mathbb{C} \backslash\{0\}$ and $f \in F$.

3.2. Definition of $\tau(N, \psi, \alpha)$. The following is a mild extension of [FK06, Proposition 2.5] and [FK08, Lemmas 6.2 and 6.3]. Most of the ideas go back to work of Turaev (cf. e.g. [Tu86] and [Tu01]).

Proposition 3.1. Let $N$ be a 3-manifold, $\psi: \pi_{1}(N) \rightarrow F$ a rationally surjective homomorphism to a free abelian group $F$ and $\alpha: \pi_{1}(N) \rightarrow G L(k, \mathbb{C})$ a representation. Then the following hold:

(1) $\Delta_{N, \psi, 0}^{\alpha} \neq 0$.

(2) If $\Delta_{N, \psi, 1}^{\alpha} \neq 0$, then $\Delta_{N, \psi, 2}^{\alpha} \neq 0$.

(3) If $\operatorname{rank}(F)>1$, then $\Delta_{N, \psi, 0}^{\alpha} \doteq 1$.

(4) If $\operatorname{rank}(F)>1$ and if $\Delta_{N, \psi, 1}^{\alpha} \neq 0$, then $\Delta_{N, \psi, 2}^{\alpha} \doteq 1$. 
If $\Delta_{N, \psi, 1}^{\alpha} \neq 0$, then we define

$$
\tau(N, \psi, \alpha):=\prod_{i=0}^{2}\left(\Delta_{N, \psi, i}^{\alpha}\right)^{(-1)^{i+1}} \in \mathbb{C}(F) .
$$

If $\Delta_{N, \psi, 1}^{\alpha}=0$, then we define $\tau(N, \psi, \alpha):=0$.

Remark. (1) Note that it is an immediate consequence of Proposition 3.1 that $\tau(N, \psi, \alpha)$ lies in $\mathbb{C}[F]$, if $\operatorname{rank}(F)>1$.

(2) Throughout this paper we identify the complex group ring of $\mathbb{Z}$ with $\mathbb{C}\left[t^{ \pm 1}\right]$, i.e. we identify $\mathbb{C}\left[t^{ \pm 1}\right]=\mathbb{C}[\mathbb{Z}]$ and $\mathbb{C}(t)=\mathbb{C}(\mathbb{Z})$. In particular if $\psi: \pi_{1}(N) \rightarrow \mathbb{Z}$ is rationally surjective, then we view $\tau(N, \psi, \alpha)$ as an element in $\mathbb{C}(t)$.

(3) The invariant $\tau(N, \psi, \alpha)$ can be viewed as a twisted Reidemeister torsion. We refer to [Mi66, Tu01, Nic03] for background on Reidemeister torsion, and we refer to [Kio96, KL99, FV10] and [FK08, Theorem 6.7] for twisted Reidemeister torsion and its relation to twisted Alexander polynomials. We will not make use of this point of view.

(4) We drop $\psi$ from the notation if $\psi$ is the projection $\pi_{1}(N) \rightarrow H_{1}(N ; \mathbb{Z}) /$ torsion, furthermore we drop $\alpha$ from the notation if $\alpha$ is the trivial one-dimensional representation.

3.3. Tensoring with one-dimensional representations. Let $N$ be a 3-manifold, let $\psi: \pi_{1}(N) \rightarrow F$ be a rationally surjective homomorphism to a free abelian group $F$ and let $\rho: F \rightarrow U(1)$ be a character. We will denote the character $\rho \circ \psi: \pi_{1}(N) \rightarrow U(1)$ by $\rho$ as well. Note that $\rho$ gives rise to a ring automorphism $\mathbb{C}[F] \rightarrow \mathbb{C}[F]$ induced by $f \mapsto \rho(f) \cdot f, f \in F$. We will denote this ring automorphism by $\rho$ as well.

The following lemma is now a straightforward consequence of the definitions:

Lemma 3.2. For any $i$ we have

$$
\Delta_{N, \psi, i}^{\rho}=\rho\left(\Delta_{N, \psi, i}\right) \in \mathbb{C}[F] .
$$

3.4. Change of variables. Let $N$ be a 3-manifold. We write $F=H_{1}(N ; \mathbb{Z}) /$ torsion. Let $\alpha: \pi_{1}(N) \rightarrow \mathrm{GL}(k, \mathbb{C})$ be a representation. Furthermore let $\phi \in H^{1}(N ; \mathbb{Z})=$ $\operatorname{Hom}(F, \mathbb{Z})$. We denote the induced ring homomorphism $\mathbb{C}[H] \rightarrow \mathbb{C}[\mathbb{Z}]=\mathbb{C}\left[t^{ \pm 1}\right]$ by $\phi$ as well. Let

$$
S=\left\{f \in \mathbb{C}[F] \mid \varphi(\mathbb{C}[F]) \neq 0 \in \mathbb{C}\left[t^{ \pm 1}\right]\right\} .
$$

Note that $\phi$ induces a homomorphism $\mathbb{C}[F] S^{-1} \rightarrow \mathbb{C}(t)$ which we also denote by $\phi$.

The following is a slight generalization of [FK08, Theorem 6.6], which in turn builds on ideas of Turaev (cf. [Tu86] and [Tu01]).

Proposition 3.3. We have $\tau(N, \alpha) \in \mathbb{C}[F] S^{-1}$, and

$$
\tau(N, \phi, \alpha) \doteq \phi(\tau(N, \alpha)) .
$$


3.5. Induced representations. Let $\pi$ be a group and let $\pi^{\prime} \subset \pi$ be a normal subgroup of index $k$. Let $\rho: \pi^{\prime} \rightarrow U(1)$ be a character. We consider the action of $\pi$ given by left multiplication on the tensor product

$$
\mathbb{C}[\pi] \otimes_{\mathbb{C}\left[\pi^{\prime}\right]} \mathbb{C}
$$

where $\pi^{\prime}$ acts on $\mathbb{C}$ via the character $\rho$. Note that $\mathbb{C}[\pi] \otimes_{\mathbb{C}\left[\pi^{\prime}\right]} \mathbb{C}$ is a complex $k$ dimensional vector space and we thus obtain a representation $\alpha: \pi \rightarrow \operatorname{GL}(k, \mathbb{C})$. This representation is called an extended character. If $\rho$ factors through a finite group, then we refer to $\alpha$ as an extended finite character. For future reference we record the following elementary fact:

Lemma 3.4. (1) An extended character is a unitary representation.

(2) An extended finite character factors through a finite group.

We conclude this section with the following well-known lemma:

Lemma 3.5. Let $N$ be a 3-manifold and let $p: M \rightarrow N$ be a finite regular covering map of index $k$. Let $\rho: \pi_{1}(M) \rightarrow U(1)$ be a character and denote by $\alpha: \pi_{1}(N) \rightarrow U(k)$ the extended character. Let $\psi: \pi_{1}(N) \rightarrow F$ be a rationally surjective homomorphism to a free abelian group $F$. Then

$$
\tau\left(M, \psi \circ p_{*}, \rho\right)=\tau(N, \psi, \alpha) .
$$

Proof. It is an immediate consequence of Shapiro's lemma (cf. [Br94, Proposition 6.2] and also [FV08] ) that for any $i$ the following equality holds:

$$
\Delta_{M, \psi \circ p_{*}, i}^{\rho}=\Delta_{N, \psi, i}^{\alpha} \in \mathbb{C}[F] .
$$

The lemma now follows from the definitions.

\section{The TWisted Alexander nORM}

4.1. Definition of the twisted Alexander norm. We now recall the definition of twisted Alexander norms introduced in [FK08. Let $N$ be a 3-manifold with $b_{1}(N)>$ 1. We write $F:=H_{1}(N ; \mathbb{Z}) /$ torsion. Let $\alpha: \pi_{1}(N) \rightarrow \mathrm{GL}(k, \mathbb{C})$ be a representation. We will now define a seminorm $y_{N}^{\alpha}$ on $H^{1}(N ; \mathbb{R})=\operatorname{Hom}(F, \mathbb{R})$ using $\tau(N, \alpha)$.

If $\tau(N, \alpha)=0$ then we set $y_{N}^{\alpha}(\phi)=0$ for any $\phi \in H^{1}(N ; \mathbb{R})$. Now suppose that $\tau(N, \alpha) \neq 0$. Since $b_{1}(N)=\operatorname{rank}(F)>1$ it follows from Proposition 3.1 that $\tau(N, \alpha) \in \mathbb{C}[F]$. We can therefore write $\tau(N, \alpha)=\sum_{f \in F} a_{f} f$ and we define

$$
\begin{aligned}
y_{N}^{\alpha}: \operatorname{Hom}(F, \mathbb{R}) & \rightarrow \mathbb{R}_{\geq 0} \\
\phi & \mapsto \max \left\{\phi\left(f_{1}\right)-\phi\left(f_{2}\right) \mid f_{1}, f_{2} \in F \text { with } a_{f_{1}} \neq 0 \text { and } a_{f_{2}} \neq 0\right\} .
\end{aligned}
$$

It is clear that $y_{N}^{\alpha}$ defines a norm on $H^{1}(N ; \mathbb{R})=\operatorname{Hom}(F, \mathbb{R})$ and we refer to $y_{N}^{\alpha}$ as the twisted Alexander norm of $(N, \alpha)$. If $\alpha$ is the trivial one-dimensional representation, then we drop $\alpha$ from the notation.

Remark. (1) With the above conventions the seminorm $y_{N}$ is just the ordinary Alexander norm introduced by McMullen $\mathrm{Mc02}$. 
(2) Twisted Alexander norms corresponding to abelian one-dimensional representations were first considered by Turaev [Tu02a], twisted Alexander norms for arbitrary representations were introduced in [FK08].

4.2. Lower bounds on the Thurston norm and fibered classes. In this section we recall results relating the Thurston norm of a 3-manifold to the twisted Alexander norms. We start out with the following lemma.

Lemma 4.1. Let $N$ be a 3-manifold with $b_{1}(N)>1$ and let $\alpha: \pi_{1}(N) \rightarrow G L(k, \mathbb{C})$ be a representation. Given any $\phi \in H^{1}(N ; \mathbb{Z})$ we have

$$
\operatorname{deg}(\tau(N, \phi, \alpha)) \leq y_{N}^{\alpha}(\phi),
$$

furthermore equality holds for all $\phi$ outside a finite collection of hyperplanes in $H^{1}(N ; \mathbb{Q})$.

The proof is essentially given in [Mc02] and we provide it for the reader's convenience as it is helpful in understanding the proof of Theorem 1.2 ,

Proof. We write $F:=H_{1}(N ; \mathbb{Z}) /$ torsion. By Proposition 3.1 we can write $\tau(N, \alpha)=$ $\sum_{f \in A} a_{f} f$ with $A \subset F$ and $a_{f} \neq 0$ for all $f \in A$. It follows from Proposition 3.3 that

$$
\tau(N, \phi, \alpha)=\phi(\tau(N, \alpha))=\sum_{f \in F} a_{f} t^{\phi(f)}
$$

It follows that

$$
\operatorname{deg}(\tau(N, \phi, \alpha))=\operatorname{deg}\left(\sum_{f \in F} a_{f} t^{\phi(f)}\right)
$$

It is clear that

$$
\operatorname{deg}\left(\sum_{f \in F} a_{f} t^{\phi(f)}\right) \leq \max \left\{\phi\left(f_{1}\right)-\phi\left(f_{2}\right) \mid f_{1}, f_{2} \in A\right\}
$$

and that equality holds unless there exist $f_{1}, f_{2} \in A$ with $\phi\left(f_{1}\right)=\phi\left(f_{2}\right)$. The lemma now follows immediately.

The following theorem is now a consequence of the above lemma, Theorem 1.1 and the fact that norms are continuous. The theorem was first proved in [FK08, Theorem 3.1].

Theorem 4.2. Let $N$ be a 3-manifold with $b_{1}(N)>1$ and let $\alpha: \pi_{1}(N) \rightarrow G L(k, \mathbb{C})$ be a representation. Then for any $\phi \in H^{1}(N ; \mathbb{R})$ we have

$$
\frac{1}{k} y_{N}^{\alpha}(\phi) \leq x_{N}(\phi)
$$

and equality holds if $\phi$ is a fibered class.

The first part of Theorem 4.2 generalizes McMullen's theorem [Mc02]. Turaev [Tu02a] proved this theorem in the special case of abelian representations. 


\section{Proof of Theorem 1.2}

5.1. Agol's virtual fibering theorem. We say that $\phi \in H^{1}(N ; \mathbb{R})$ is quasi-fibered if there exists a fibered face $F$ of the Thurston norm ball such that $\phi$ lies in a cone on the closure of $F$. Put differently, $\phi$ is quasi-fibered if and only if any neighborhood of $\phi$ in $H^{1}(N ; \mathbb{R})$ contains a fibered class. Note that in particular fibered classes are quasi-fibered.

We recall from Ag08 the following definition: A group $\pi$ is residually finite rationally solvable (RFRS) if there exists a filtration of groups $\pi=\pi_{0} \supset \pi_{1} \supset \pi_{2} \ldots$ such that the following hold:

(1) $\bigcap_{i} \pi_{i}=\{1\}$;

(2) $\pi_{i}$ is a normal, finite-index subgroup of $\pi$ for any $i$;

(3) for any $i$ the map $\pi_{i} \rightarrow \pi_{i} / \pi_{i+1}$ factors through $\pi_{i} \rightarrow H_{1}\left(\pi_{i} ; \mathbb{Z}\right) /$ torsion.

We refer to Ag08 for details and more information on RFRS groups. A group is virtually RFRS if it admits a finite index subgroup that is RFRS.

We can now formulate Agol's virtual fibering theorem (see Ag08) which is one of the key ingredients in our proof of Theorem 1.2 .

Theorem 5.1. (Agol) Let $N$ be an irreducible 3-manifold such that $\pi_{1}(N)$ is virtually $R F R S$. Then given any $\phi \in H^{1}(N ; \mathbb{R})$ there exists a finite regular cover $p: M \rightarrow N$, such that $p^{*} \phi \in H^{1}(M ; \mathbb{R})$ is quasi-fibered.

The following is a well-known consequence of Agol's virtual fibering theorem:

Corollary 5.2. Let $N$ be an irreducible 3-manifold with virtually RFRS fundamental group. Then there exists a finite regular cover $p: M \rightarrow N$ such that for every nontrivial class $\phi \in H^{1}(N ; \mathbb{R})$, the class $p^{*} \phi \in H^{1}(M ; \mathbb{R})$ is quasi-fibered.

Proof. Let $\phi \in H^{1}(N ; \mathbb{R})$ be a class contained in the cone over a top-dimensional open face of $B(N)$. By Agol's virtual fibering theorem there exists a finite regular cover $p: M \rightarrow N$ such that $p^{*} \phi$ is quasi-fibered. By (2) the pull back map $p^{*}: H^{1}(N ; \mathbb{R}) \rightarrow$ $H^{1}(M ; \mathbb{R})$ is, up to scale, a monomorphism of normed vector spaces when we endow these spaces with their respective Thurston norm. It follows that the pull back under $p$ of any class in the closure of the open cone (in $H^{1}(N ; \mathbb{R})$ ) determined by $\phi$ will be quasi-fibered in $M$. For the same reason if a class lies in the closure of a fibered cone, its pull back under further finite covers will enjoy the same property (since pullbacks of fibrations are fibrations). Recall now that the Thurston norm ball of a 3-manifold is a finite, convex polyhedron, in particular it has finitely many top-dimensional open faces. By picking one class in the cone above each of these faces, and repeatedly applying Agol's theorem to the (transfer of) each such class, we obtain after finitely many steps a finite regular cover, that we will denote as well by $p: M \rightarrow N$, such that $p^{*} \phi$ is quasi-fibered for any $\phi \in H^{1}(N ; \mathbb{R})$. By going to a further cover, if necessary, we can arrange that $M$ is in fact a finite regular cover of $N$. 
5.2. The results of Agol, Liu, Przytycki-Wise and Wise. The 'virtually RFRS' condition in Theorem 5.1 might at a first glance look very restrictive. It is thus amazing that over the last few years it was shown that 'most' 3-manifold groups are in fact virtually RFRS.

The key in proving that 3-manifold groups are virtually RFRS is the notion of a 'virtually special' group introduced by Haglund and Wise [HW08. The precise definition of 'virtually special' is of no concern to us. The only thing we need is the following theorem of Haglund and Wise [HW08] and Agol Ag08, Theorem 2.2]:

Theorem 5.3. Let $\pi$ be a group which is virtually special, then $\pi$ is virtually $R F R S$.

We can now formulate the following theorem which was announced by Wise Wi09] in 2009, with the details of the proof being provided in Wi12 (see also [Wi11]).

Theorem 5.4. (Wise) Let $N$ be a hyperbolic 3-manifold such that one of the following holds:

(1) $b_{1}(N)>1$, or

(2) $b_{1}(N)=1$ and $N$ is not fibered,

(3) $N$ has non-trivial boundary,

then $\pi_{1}(N)$ is virtually special.

Remark. We give now precise references for the theorem:

(1) Let $N$ be a closed hyperbolic 3-manifold which admits a geometrically finite surface. Theorem 14.1 of [Wi12] asserts that $\pi_{1}(N)$ is 'virtually special', which by work of implies that $\pi_{1}(N)$ is virtually RFRS. Thurston and Bonahon Bo86] showed that an incompressible connected surface $\Sigma \subset N$ either $\Sigma$ lifts to a surface fiber in a finite cover, or $\Sigma$ is geometrically finite. It now follows from basic arguments that a closed hyperbolic 3-manifold with either

(a) $b_{1}(N)>1$, or

(b) $b_{1}(N)=1$ and $N$ not fibered,

admits a geometrically finite surface. By the above this implies that the fundamental group of such a hyperbolic 3-manifold is virtually RFRS.

(2) If $N$ has boundary, then the statement of Theorem 5.4 is precisely Theorem 16.28 together with Corollary 14.16 of [Wi12].

(3) Agol Ag12, building on the Surface Subgroup Theorem of Kahn-Markovic KM12] and on work of Wise [Wi12] showed that in fact the fundamental group of any closed hyperbolic 3-manifold is virtually RFRS. We will not make use of this result since in our situation it suffices to consider hyperbolic 3-manifolds which satisfy the conditions of Theorem 5.4. Also note that Agol's result does not cover the case of hyperbolic 3-manifolds with non-trivial toroidal boundary.

The following theorem was proved by Liu [Liu11, Theorem 1.1]. 
Theorem 5.5. (Liu) Let $N$ be a graph manifold which supports a non-positively curved metric. Then $\pi_{1}(N)$ is virtually special.

Note that by [Le95] any graph manifold with non-trivial boundary is non-positively curved. For such graph manifolds the theorem was also proved by Przytycki-Wise PW11.

The following theorem is also due to Przytycki-Wise.

Theorem 5.6. (Przytycki-Wise) Let $N$ be a 3-manifold which admits a nontrivial JSJ decomposition with at least one hyperbolic piece. Then $\pi_{1}(N)$ is virtually special.

5.3. Properties of the twisted Alexander norm. Let $F$ be an free abelian group and let $\rho: F \rightarrow U(1)$ be a character. Note that $\rho$ gives rise to a ring homomorphism $\mathbb{C}[F] \rightarrow \mathbb{C}$ which we also denote by $\rho$.

Lemma 5.7. Let $F$ be a free abelian group and let $\left\{p_{i}=\sum_{j=1}^{d_{i}} a_{i j} f_{i j} \in \mathbb{C}[F], i=\right.$ $1, \ldots, l\}$ be a collection of non-zero polynomials. Then there exists a character $\rho: F \rightarrow U(1) \subset \mathbb{C}$ which factors through a finite group such that all $\sum_{j=1}^{d_{i}} a_{i j} \rho\left(f_{i j}\right) \in \mathbb{C}$ are simultaneously non-zero.

Proof. Given $i \in\{1, \ldots, l\}$ it is clear that

$$
V_{i}:=\left\{\kappa \in \operatorname{Hom}(F, \mathbb{Q}) \mid \kappa\left(f_{i j}\right)=\kappa\left(f_{i k}\right) \text { for some } j \neq k, a_{i j} a_{i k} \neq 0\right\}
$$

is a finite union of codimension one subspaces of $\operatorname{Hom}(F, \mathbb{Q})$. We can now pick an epimorphism $\kappa: F \rightarrow \mathbb{Z}$ such that $\kappa \notin V_{1} \cup \cdots \cup V_{l}$. For all $z \in U(1) \subset \mathbb{C}$ we now consider the character

$$
\begin{aligned}
\kappa_{z}: F & \rightarrow U(1) \\
f & \mapsto z^{\kappa(f)} .
\end{aligned}
$$

Since $\kappa \notin V_{1} \cup \cdots \cup V_{l}$, the polynomials $q_{i}(t)=\sum_{j=1}^{d_{i}} a_{i j} t^{\kappa\left(f_{i j}\right)} \in \mathbb{C}\left[t^{ \pm 1}\right]$ are non-zero. It is clear that there exists a root of unity $z$ with $q_{i}(z) \neq 0 \in \mathbb{C}$ for $i=1, \ldots, l$. In correspondence of such $z \in U(1)$ the character $\rho:=\kappa_{z}$ has the desired property.

Proposition 5.8. Let $M$ be a 3-manifold. Then there exists a character $\rho: \pi_{1}(M) \rightarrow$ $U(1)$ which factors through a finite group such that

$$
y_{M}(\phi)=\operatorname{deg}(\tau(M, \phi, \rho))
$$

for any $\phi \in H^{1}(M ; \mathbb{Z})$.

Proof. We write $F:=H_{1}(M ; \mathbb{Z}) /$ torsion. If $\operatorname{rank}(F)=1$ or if $\tau(M)=0$, then there is nothing to prove. We thus consider the case $\operatorname{rank}(F)>1$ and $\tau(M) \neq 0$. By Proposition 3.1 we have $\tau(M)=\Delta_{M} \in \mathbb{C}[F]$. We now write

$$
\Delta_{M}=\sum_{f \in F} a_{f} f
$$


with $a_{f} \in \mathbb{C}, f \in F$. Given a subset $A \subset F \otimes \mathbb{R}$ we write

$$
\Delta_{A}:=\sum_{f \in F \cap A} a_{f} f \in \mathbb{C}[F]
$$

We say $\Delta_{A} \in \mathbb{C}[F]$ is a face polynomial of $\Delta_{M}$ if $A \subset N\left(\Delta_{M}\right)$ is a face of the Newton polyhedron of $\Delta_{M}$.

We denote by $\Delta_{A_{1}}, \ldots, \Delta_{A_{l}}$ the face polynomials of $\Delta_{M}$. By Lemma 5.7 there exists a character $\rho: F \rightarrow U(1)$ factoring through a finite group such that

$$
\sum_{f \in F \cap A_{1}} a_{f} \rho(f), \ldots, \sum_{f \in F \cap A_{l}} a_{f} \rho(f)
$$

are simultaneously non-zero complex numbers.

Claim. Let $\phi \in H^{1}(M ; \mathbb{Z})=\operatorname{Hom}(F, \mathbb{Z})$, then $y_{M}(\phi)=\operatorname{deg}(\tau(M, \phi, \rho))$.

First note that $\phi$ extends to a homomorphism $\operatorname{Hom}(F \otimes \mathbb{R}, \mathbb{R})$ which we also denote by $\phi$. There exist maximal faces $A_{t}$ and $A_{b}$ of $N\left(\Delta_{M}\right)$, such that $\phi$ takes on maximal values on $A_{t} \subset F \otimes \mathbb{R}$ and minimal values on $A_{b} \subset F \otimes \mathbb{R}$. Put differently, $\phi$ is constant on $A_{t}$ and $A_{b}$, and

$$
\phi\left(A_{b}\right) \leq \phi(f) \leq \phi\left(A_{t}\right)
$$

for any $N\left(\Delta_{M}\right)$ and equalities hold only if $f \in A_{b}$ respectively $f \in A_{t}$. Recall that

$$
\sum_{f \in F \cap A_{b}} a_{f} \rho(f) \neq 0 \in \mathbb{C} \text { and } \sum_{f \in F \cap A_{t}} a_{f} \rho(f) \neq 0 \in \mathbb{C} .
$$

At this point, applying Lemma 3.2 and Proposition 3.3 we have

$$
\tau(M, \phi, \rho)=\sum_{f \in F} a_{f} \rho(f) t^{\phi(f)}
$$

It follows from (3) and the above discussion that

$$
\begin{aligned}
\text { highest degree term of } \tau(M, \phi, \rho) \in \mathbb{C}\left[t^{ \pm 1}\right] & =\left(\sum_{f \in F \cap A_{t}} a_{f} \rho(f)\right) t^{\phi\left(A_{t}\right)} \text {, and } \\
\text { lowest degree term of } \tau(M, \phi, \rho) \in \mathbb{C}\left[t^{ \pm 1}\right] & =\left(\sum_{f \in F \cap A_{b}} a_{f} \rho(f)\right) t^{\phi\left(A_{b}\right)},
\end{aligned}
$$

hence

$$
\begin{aligned}
y_{M}(\phi) & =\max \left\{\phi\left(f_{1}\right)-\phi\left(f_{2}\right) \mid f_{1}, f_{2} \in F \text { with } a_{f_{1}} \neq 0 \text { and } a_{f_{2}} \neq 0\right\} \\
& =\phi\left(A_{t}\right)-\phi\left(A_{b}\right) \\
& =\operatorname{deg}(\tau(M, \phi, \rho)) .
\end{aligned}
$$

This concludes the proof of the claim. 
5.4. Proof of Theorem 1.2. The following theorem, together with Lemma 3.4, clearly implies Theorem 1.2 ,

Theorem 5.9. Let $N$ be an irreducible 3-manifold which is not a closed graph manifold. Then there exists an extended finite character $\alpha: \pi_{1}(N) \rightarrow U(k)$ such that

$$
\frac{1}{k} \operatorname{deg}(\tau(N, \phi, \alpha))=x_{N}(\phi) \text { for any } \phi \in H^{1}(N ; \mathbb{Z}) .
$$

Proof. Let $N$ be an irreducible 3-manifold which is not a closed graph manifold. If $b_{1}(N)=0$, then there is nothing to prove. If $N$ is a closed hyperbolic fibered 3manifold with $b_{1}(N)=1$, then the trivial representation has the required property. For all the remaining cases, it follows from Theorems 5.3, 5.4, 5.5 and 5.6 that $\pi_{1}(N)$ is virtually RFRS. By Corollary 5.2 there exists a regular finite cover $p: M \rightarrow N$ such that $p^{*} \phi$ is quasi-fibered for any $\phi \in H^{1}(N ; \mathbb{R})$. We denote by $k$ the order of the cover. It follows from Theorem 4.2 and from the continuity of $y_{M}$ and $x_{M}$ that $y_{M}(\psi)=x_{M}(\psi)$ for any quasi-fibered class $\psi \in H^{1}(M ; \mathbb{R})$. In particular we obtain that

$$
y_{M}\left(p^{*} \phi\right)=x_{M}\left(p^{*} \phi\right) \text { for any } \phi \in H^{1}(N ; \mathbb{R}) \text {. }
$$

By Proposition 5.8 there exists a character $\rho: \pi_{1}(M) \rightarrow U(1)$ which factors through a finite group such that

$$
y_{M}(\psi)=\operatorname{deg}(\tau(M, \psi, \rho))
$$

for any $\psi \in H^{1}(M ; \mathbb{Z})$. We now denote by $\alpha: \pi_{1}(N) \rightarrow U(k)$ the corresponding extended finite character.

Now let $\phi \in H^{1}(N ; \mathbb{Z})$. Lemma 3.5 implies

$$
\tau\left(M, p^{*} \phi, \rho\right)=\tau(N, \phi, \alpha)
$$

It now follows from Equalities (2), (44), (15) and (6) and from Theorem 1.1 that

$$
\begin{aligned}
x_{N}(\phi) & =\frac{1}{k} x_{M}\left(p^{*} \phi\right) \\
& =\frac{1}{k} y_{M}\left(p^{*} \phi\right) \\
& =\frac{1}{k} \operatorname{deg}\left(\tau\left(M, p_{*} \phi, \rho\right)\right)=\frac{1}{k} \operatorname{deg}(\tau(N, \phi, \alpha)) \leq x_{N}(\phi) .
\end{aligned}
$$

We conclude that

$$
\frac{1}{k} \operatorname{deg}(\tau(N, \phi, \alpha))=x_{N}(\phi) \text { any } \phi \in H^{1}(N ; \mathbb{Z})
$$

We can also reinterpret Theorem 1.2 in terms of twisted Alexander norms:

Corollary 5.10. Let $N$ be an irreducible 3-manifold which is not a closed graph manifold. Then there exists an extended finite character $\alpha: \pi_{1}(N) \rightarrow U(k)$ such that

$$
\frac{1}{k} y_{N}^{\alpha}(\phi)=x_{N}(\phi) \text { for any } \phi \in H^{1}(N ; \mathbb{R}) \text {. }
$$


Proof. Let $N$ be an irreducible 3-manifold which is not a closed graph manifold. By Theorem 5.9 there exists an extended finite character $\alpha: \pi_{1}(N) \rightarrow U(k)$ such that

$$
\frac{1}{k} \operatorname{deg}(\tau(N, \phi, \alpha))=x_{N}(\phi) \text { for any } \phi \in H^{1}(N ; \mathbb{Z}) .
$$

Lemma 4.1 and Theorem 4.2 imply that

$$
x_{N}(\phi)=\frac{1}{k} \operatorname{deg}(\tau(N, \phi, \alpha)) \leq \frac{1}{k} y_{N}^{\alpha}(\phi) \leq x_{N}(\phi),
$$

hence for any $\phi \in H^{1}(N ; \mathbb{Z})$ we have

$$
\frac{1}{k} y_{N}^{\alpha}(\phi)=x_{N}(\phi)
$$

It now follows from the linearity and the continuity of the norms $y_{N}^{\alpha}$ and $x_{N}$ that this equality holds in fact for all real classes.

\section{An Algorithm FOR Determining the Thurston NORM}

Let $N$ be an irreducible 3-manifold which is not a closed graph manifold. We will now show the following:

(A) Given $\phi \in H^{1}(N ; \mathbb{Z})$ Theorem 5.9 gives rise to an algorithm $A$ which determines the Thurston norm of $\phi$.

(B) Corollary 5.10 gives rise to an algorithm $B$ which determines the Thurston norm of $N$.

Since the former algorithm is much easier to explain we treat it separately, even though of course the second algorithm is stronger than the first algorithm. We explain the algorithms in a somewhat informal way, we leave it to the reader to formulate a completely formal algorithm.

6.1. Extended finite characters. Let $\pi$ be a finitely presented group. We can then systematically go through all homomorphisms from $\pi$ to all permutation groups. Since every finite group is a subgroup of a permutation group we can thus go through all epimorphisms to finite group. For each epimorphism $\alpha: \pi \rightarrow G$ onto a finite group we can determine $H_{1}(\operatorname{Ker}(\alpha) ; \mathbb{Z})$ using the Reidemeister-Schreier method. It is now straightforward to see that one can systematically find a sequence of extended finite characters, such that up to conjugation every finite extended character will eventually appear.

Put differently, we can inductively define extended finite characters $\alpha_{i}, i \in \mathbb{N}$, such that given any extended finite character $\alpha$ there exists an $i$, such that $\alpha$ and $\alpha_{i}$ are conjugate. 
6.2. Algorithm $A$. Let $N$ be an irreducible 3-manifold which is not a closed graph manifold and let $\phi \in H^{1}(N ; \mathbb{Z})$.

The algorithm $A$ consists of two programs $P(\phi)$ and $Q(\phi)$ running at the same time:

(1) For $i=1,2,3, \ldots$ Program $P(\phi)$ computes $\tau\left(N, \phi, \alpha_{i}\right) \in \mathbb{C}(t)$. Note that $\tau\left(N, \phi, \alpha_{i}\right)$ can be calculated efficiently using Fox calculus (see e.g. [FK06]).

(2) Program $Q(\phi)$ lists all properly embedded surfaces dual to $\phi$ up to isotopy and computes their complexities. Such a program can for example be written using normal surfaces (see e.g. [CT09]).

It follows from Theorem 5.9 that after finitely many steps the lower bound on the Thurston norm coming from $P(\phi)$ agrees with the upper bound on the Thurston norm coming from $Q(\phi)$.

This algorithm sounds inefficient, but the calculations in [FK06] and [DFJ11] show that in practice twisted Alexander polynomials are very efficient at determining the Thurston norm for a given class $\phi$.

6.3. Algorithm $B$. Before we explain the algorithm for determining the Thurston norm ball we state the following elementary lemma:

Lemma 6.1. Let $x$ and $y$ be seminorms on a real vector space $V$ with $y \leq x$. Denote by $B$ and $C$ the norm balls of $x$ and $y$. Let $\phi_{1}, \ldots, \phi_{k}$ be a set of non-zero classes in $V$ such that each open cone on a top dimensional face of $C$ contains at least one $\phi_{i}$. If $x\left(\phi_{i}\right)=y\left(\phi_{i}\right)$ for $i=1, \ldots, k$, then $x=y$ for all $\phi \in V$.

Let $N$ be an irreducible, triangulated 3-manifold which is not a closed graph manifold. Recall that we denote the Thurston norm ball of $N$ by

$$
B(N):=\left\{\phi \in H^{1}(N ; \mathbb{Q}) \mid x_{N}(\phi) \leq 1\right\} .
$$

Given a representation $\alpha: \pi_{1}(N) \rightarrow \mathrm{GL}(k, \mathbb{C})$ we also write

$$
B(N, \alpha):=\left\{\phi \in H^{1}(N ; \mathbb{Q}) \mid \frac{1}{k} y_{N}^{\alpha}(\phi) \leq 1\right\} .
$$

Note that Theorem 4.2 says that $B(N, \alpha) \subset B(N)$. Also note that $B(N, \alpha)$ is the dual to the Newton polygon of $\tau(N, \alpha)$, in particular the vertices of $B(N, \alpha)$ can be determined easily using $\tau(N, \alpha)$.

We finally write $\Phi:=\emptyset \subset H^{1}(N ; \mathbb{Z})$ and we denote by $z$ the zero norm. The algorithm now consists of two programs running at the same time:

(1) If $\phi_{1}, \ldots, \phi_{k}$ denote the elements of $\Phi$, then we apply algorithms $Q\left(\phi_{1}\right), \ldots, Q\left(\phi_{k}\right)$ from the previous section and we compute the complexities of the corresponding surfaces.

(2) For $i=1,2, \ldots$ Program $P$ computes $\tau\left(N, \alpha_{i}\right)$ and determines $B\left(N, \alpha_{i}\right)$. If

$$
B\left(N, \alpha_{j}\right) \subsetneq B\left(N, \alpha_{i}\right) \text { for } j=1, \ldots, i-1,
$$


then we denote by $z$ the norm $y_{N}^{\alpha_{i}}$ and for each open cone on a top dimensional face of $B\left(N, \alpha_{i}\right)$ we pick a class in $H^{1}(N ; \mathbb{Z})$ and we denote the resulting set by $\Phi$ and we restart (1).

We terminate the two programs when the complexities of $C\left(\phi_{1}\right), \ldots, C\left(\phi_{k}\right)$ agree with $z\left(\phi_{1}\right), \ldots, z\left(\phi_{k}\right)$. It then follows from Lemma 6.1 that $z$ equals the Thurston norm on $N$.

It remains to show that this algorithm terminates after finitely many steps. First note that by Corollary 5.10 there exists an $i$ such that $B\left(N, \alpha_{i}\right)=B(N)$. After finitely many further steps the first program will find Thurston norm surfaces representing $\phi_{1}, \ldots, \phi_{k}$. The program terminates at this point.

\section{REFERENCES}

[Ag08] I. Agol, Criteria for virtual fibering, Journal of Topology 1: 269-284 (2008)

[Ag12] I. Agol, The virtual Haken conjecture, with an appendix by I. Agol, D. Groves, J. Manning, preprint (2012).

[Bo86] F. Bonahon, Bouts des variétés hyperboliques de dimension 3, Ann. of Math. (2), 124 (1986), $71-158$.

[Br94] K. S. Brown, Cohomology of groups, Graduate Texts in Mathematics, 87. Springer-Verlag, New York, 1994.

[Ch03] J. Cha, Fibred knots and twisted Alexander invariants, Transactions of the AMS 355: 4187$4200(2003)$

[CT09] S. Tillmann and D. Cooper, The Thurston norm via normal surfaces, Pacific Journal of Mathematics 239 (2009) 1-15.

[DFJ11] N. Dunfield, S. Friedl and N. Jackson, Twisted Alexander polynomials of hyperbolic knots, preprint (2011), to appear in Experimental Mathematics

[FK06] S. Friedl and T. Kim, The Thurston norm, fibered manifolds and twisted Alexander polynomials, Topology 45, 929-953 (2006)

[FK08] S. Friedl and T. Kim, Twisted Alexander norms give lower bounds on the Thurston norm, Trans. Amer. Math. Soc. 360 (2008), 4597-4618

[FSW12] S. Friedl, S. Williams and D. Silver, Complexities in groups, 2-complexes and 3-manifolds, in preparation (2012)

[FV08] S. Friedl and S. Vidussi, Twisted Alexander polynomials and symplectic structures, Amer. J. Math. 130, no 2: 455- 484 (2008)

[FV10] S. Friedl and S. Vidussi, A survey of twisted Alexander polynomials, The Mathematics of Knots: Theory and Application (Contributions in Mathematical and Computational Sciences), editors: Markus Banagl and Denis Vogel (2010), p. 45-94.

[FV11a] S. Friedl and S. Vidussi, Twisted Alexander polynomials detect fibered 3-manifolds, Annals of Math. 173 (2011), 1587-1643

[FV11b] S. Friedl and S. Vidussi, Twisted Alexander polynomials and fibered 3-manifolds, LowDimensional and Symplectic Topology, Proc. Sympos. Pure Math. 82 (2011), 111-130.

[FV11c] S. Friedl and S. Vidussi, A vanishing theorem for twisted Alexander polynomials with applications to symplectic 4-manifolds, Preprint (2011)

[FV11d] S. Friedl and S. Vidussi, Minimal Genus on 4-manifolds with a Free Circle Action, preprint (2011)

[Ga83] D. Gabai, Foliations and the topology of 3-manifolds, J. Differential Geometry 18, no. 3: 445-503 (1983) 
[GKM05] H. Goda, T. Kitano and T. Morifuji, Reidemeister Torsion, Twisted Alexander Polynomial and Fibred Knots, Comment. Math. Helv. 80, no. 1: 51-61 (2005)

[GM03] H. Goda and T. Morifuji, Twisted Alexander polynomial for SL $(2, \mathbb{C})$-representations and fibered knots, C. R. Math. Acad. Sci. Soc. R. Can. 25 (2003), no. 4, 97-101

[HW08] F. Haglund and D. Wise, Special cube complexes, Geom. Funct. Anal. 17, No. 5, 1551-1620 (2008)

[KM12] J. Kahn and V. Markovic, Immersing almost geodesic surfaces in a closed hyperbolic three manifold, Ann. of Math. 175 (2012), 1127-1190.

[KL99] P. Kirk and C. Livingston, Twisted Alexander invariants, Reidemeister torsion and CassonGordon invariants, Topology 38 (1999), no. 3, 635-661.

[Kio96] T. Kitano, Twisted Alexander polynomials and Reidemeister torsion, Pacific J. Math. 174 (1996), no. 2, 431-442.

[Kiy08] T. Kitayama, Normalization of twisted Alexander invariants, Preprint (2008)

[Le95] B. Leeb, 3-manifolds with(out) metrics of nonpositive curvature, Invent. Math. 122 (1995), $277-289$.

[Lin01] X. S. Lin, Representations of knot groups and twisted Alexander polynomials, Acta Math. Sin. (Engl. Ser.) 17, no. 3: 361-380 (2001)

[Liu11] Y. Liu, Virtual cubulation of nonpositively curved graph manifolds, Preprint (2011)

[Mc02] C. T. McMullen, The Alexander polynomial of a 3-manifold and the Thurston norm on cohomology, Ann. Sci. Ecole Norm. Sup. (4) 35, no. 2: 153-171 (2002)

[Mi66] J. Milnor, Whitehead torsion, Bull. Amer. Math. Soc. 72 (1966), 358-426.

[Nic03] L. Nicolaescu, The Reidemeister torsion of 3-manifolds, de Gruyter Studies in Mathematics, 30. Walter de Gruyter \& Co., Berlin, 2003.

[Pa07] A. Pajitnov, Novikov homology, twisted Alexander polynomials, and Thurston cones, St. Petersburg Math. J. 18 (2007), no. 5, 809-835

[PW11] P. Przytycki and D. Wise, Graph manifolds with boundary are virtually special, Preprint (2011)

[PW12] P. Przytycki and D. Wise, Mixed 3-manifolds are virtually special, Preprint (2012).

[St62] J. Stallings, On fibering certain 3-manifolds, 1962 Topology of 3-manifolds and related topics (Proc. The Univ. of Georgia Institute, 1961) pp. 95-100 Prentice-Hall, Englewood Cliffs, N.J. (1962)

[Ti70] D. Tischler, On fibering certain foliated manifolds over $S^{1}$, Topology 9, 153-154 (1970)

[Th86] W. P. Thurston, A norm for the homology of 3-manifolds, Mem. Amer. Math. Soc. 339: 99-130 (1986).

[Tu86] V. Turaev, Reidemeister torsion in knot theory, Russian Math. Surveys 41 (1986), no. 1, 119-182.

[Tu01] V. Turaev, Introduction to combinatorial torsions, Birkhäuser, Basel, (2001)

[Tu02a] V. Turaev, Torsions of 3-manifolds, Progress in Mathematics, 208. Birkhäuser Verlag, Basel, 2002.

[Tu02b] V. Turaev, A norm for the cohomology of 2-complexes, Algebraic \& Geometric Topology 2 (2002) $137-155$

[Wa94] M. Wada, Twisted Alexander polynomial for finitely presentable groups, Topology 33, no. 2: 241-256 (1994)

[Wi09] D. Wise, The structure of groups with a quasiconvex hierarchy, Electronic Res. Ann. Math. Sci., Volume 16, Pages 44-55 (2009)

[Wi11] D. Wise, From riches to RAAGs: 3-manifolds, right-angled Artin groups, and cubical geometry, lecture notes for the NSF-CBMS conference August 1-5, 2011 held at the CUNY Graduate Center, 78 pages (2011) 
[Wi12] D. Wise, The structure of groups with a quasiconvex hierarchy, 181 pages, Preprint (2012) downloaded on April 16, 2012 from the webpage for the NSF-CBMS conference.

Mathematisches Institut, Universität ZU KöLn, Germany

E-mail address: sfriedl@gmail.com

Department of Mathematics, University of California, Riverside, CA 92521, USA

E-mail address: svidussi@math.ucr.edu 